IJMMS 25:4 (2001) 253-263

PII. S0161171201003507

http://ijmms.hindawi.com

(c) Hindawi Publishing Corp.

\title{
NEW INVERSION FORMULAS FOR THE KRÄTZEL TRANSFORMATION
}

\author{
DOMINGO ISRAEL CRUZ-BÁEZ and JOSÉ RODRÍGUEZ EXPÓSITO
}

(Received 9 June 1999)

\begin{abstract}
We study in distributional sense by means of the kernel method an integral transform introduced by Krätzel. It is well known that the cited transform generalizes to the Laplace and Meijer transformation. Properties of analyticity, boundedness, and inversion theorems are established for the generalized transformation.
\end{abstract}

2000 Mathematics Subject Classification. Primary 44A15, 46F12.

1. Introduction. In this paper, we study the following integral transform,

$$
\left(K_{v}^{\rho} f\right)(x)=\int_{0}^{\infty} Z_{\rho}^{v}(x y) f(y) d y, \quad x>0,
$$

where $Z_{\rho}^{v}(x)$ denotes the function

$$
Z_{\rho}^{v}(x)=\int_{0}^{\infty} t^{\nu-1} e^{-t^{\rho}-x / t} d t
$$

with $\rho>0, \rho \in \mathbb{N}, v \in \mathbb{C}$. The $K_{v}^{\rho}$ transform is reduced to the Meijer transform when $\rho=1$ and to the Laplace transform when $\rho=1$ and $v= \pm 1 / 2$. Zemanian realized a wide study of the Laplace and Meijer transformations, in distribution spaces (cf. [12, 13]). Later, Krätzel, introduced the $K_{v}^{\rho}$ transformation, which generalizes the Meijer transform, and in a series of papers, investigated it in the classical sense (see [5, 6]). In [8], Rao and Debnath investigated the $K_{v}^{\rho}$ transformation on certain spaces of distributions by means of the kernel method. Recently, the cited transformation is studied in [1] by the adjoint method on the McBride's spaces.

In [3], the study of Krätzel is continued obtaining Abelian, Tauberian theorems, and some inversion formulas in the classical sense. Moreover, we can emphasize in [2] the work developed by the authors studying the $K_{v}^{\rho}$ transform on weighted $L^{p}$ spaces, improving a result of [4].

Motivated by the cited papers, we accomplish a study of the $K_{v}^{\rho}$ transform by means of the kernel method on the space of distributions of compact support.

By $\mathfrak{E}(I)$ we denote the infinitely differentiable functions $\phi(t), t \in I=(0, \infty)$, such that for all compact $K$ we have

$$
\gamma_{k}(\phi)=\sup _{t \in K}\left|\frac{d^{k}}{d t^{k}} \phi(t)\right|<\infty,
$$

for every $k \in \mathbb{N}$. By $\mathbb{E}^{\prime}(I)$ we denote the dual space of $\mathbb{E}(I)$. Moreover, by $\mathbb{D}(I)$, 逼 $(I)$ denote the spaces of functions and distributions that can be found in $[9,11]$. 
Consider the following useful properties.

In [1] we see that

$$
\frac{d^{k}}{d x^{k}} Z_{\rho}^{v}(x)=(-1)^{k} Z_{\rho}^{v-k}(x)
$$

By (1.4) and the asymptotic behaviour of $Z_{\rho}^{\nu}(x)$, we deduce that for certain positive constant $\alpha_{1}$ and $k \in \mathbb{N}$, we have

$$
\left|\frac{d^{k}}{d x^{k}}\left(Z_{\rho}^{v}(x)\right)\right| \leq \alpha_{1} \cdot x^{(2 v-\rho) /(2 \rho+2)} e^{-x^{\rho /(\rho+1)}},
$$

for all $x \in K, K$ compact and $v \in \mathbb{C}$.

Moreover, by [5] we have that if $\rho \in \mathbb{N}$,

$$
A_{\rho, v} Z_{\rho}^{v}(x)=(-1)^{\rho} Z_{\rho}^{v}(x)
$$

where

$$
A_{\rho, v}=-\rho^{-1} \cdot x^{\nu-\rho+1} \frac{d}{d x} x^{\rho-v} \frac{d^{\rho}}{d x^{\rho}} .
$$

On the other hand, $B_{\rho, v, k}$ denotes $B_{\rho, v, k}=C_{1} \cdot y^{-\rho k-1} B_{\rho, v}^{k}$, where

$$
\begin{aligned}
B_{\rho, v} & =x^{-v+\rho+1} \frac{d}{d x} x^{\rho+v} \frac{d^{\rho}}{d x^{\rho}}, \\
C_{1} & =\frac{(\rho k)^{\rho k-(2 v-\rho) /(2 \rho+2)-1 /(\rho+1)+1} \rho}{\Gamma\left(\rho k-\frac{2 v-\rho}{2 \rho+2}-\frac{1}{\rho+1}+1\right) \Gamma\left(\frac{\rho k-(2 v-\rho) /(2 \rho+2)-1 /(\rho+1)+1+v}{\rho}\right)} .
\end{aligned}
$$

The Mellin transform is defined by $2 \mathfrak{b}\{f\}(s)=\int_{0}^{\infty} x^{s-1} f(x) d x$ and the Mellin transform of the kernel is given by

$$
2 \mathfrak{W}\left(Z_{\rho}^{v}(x)\right)(s)=\frac{1}{\rho} \Gamma(s) \Gamma\left(\frac{s+v}{\rho}\right),
$$

if $\operatorname{Re} s+\min (0, \operatorname{Re} v)>0$.

2. The generalized $K_{v}^{\rho}$-transform on $\mathfrak{E}^{\prime}(I)$. Let $v \in \mathbb{C}$ and $\rho \in \mathbb{N}$. For every $f \in$ $\mathfrak{E}^{\prime}(I)$ we define the generalized $K_{v}^{\rho}$ transform by the relation

$$
F(x)=\left(K_{v}^{\rho} f\right)(x)=\left\langle f(t), Z_{\rho}^{v}(x t)\right\rangle .
$$

It is easy to see that $Z_{\rho}^{v}(x t) \in \mathfrak{E}(I)$ for $x$ fixed, $x>0$. Furthermore, if $f$ is a locally absolutely integrable function, then the generalized transform of $f$ is reduced to the classic $K_{v}^{\rho}$ transform.

Proposition 2.1. The operators $A_{\rho, v}$ and $B_{\rho, v}$ define a continuous linear mapping from $\in(I)$ into itself.

Proof. It is established without difficulty that

$$
\gamma_{k}\left(A_{\rho, v} \phi\right) \leq C \cdot \sum_{j=1}^{\rho+1} \gamma_{k+j}(\phi), \quad \gamma_{k}\left(B_{\rho, v} \phi\right) \leq C \cdot \sum_{j=1}^{\rho+1} \gamma_{k+j}(\phi),
$$

for every $\phi \in \mathfrak{E}(I)$. 
We define the generalized operator $A_{\rho, v}^{*}$ on $\mathfrak{E}^{\prime}(I)$ as the adjoint operator of $A_{\rho, v}$, that is,

$$
\left\langle A_{\rho, v}^{*} f, \phi\right\rangle=\left\langle f, A_{\rho, v} \phi\right\rangle,
$$

with $f \in \mathfrak{E}^{\prime}(I)$ and $\phi \in \mathfrak{E}(I)$. Moreover, by Proposition 2.1, $A_{\rho, v}^{*}$ is a continuous linear mapping from $\mathbb{E}^{\prime}(I)$ into itself. Note that the same occurs for the operator $B_{\rho, v}$.

Next are established some properties of the generalized $K_{v}^{\rho}$ transformation.

Proposition 2.2. Let $f \in \mathfrak{E}^{\prime}(I)$. If $F(x)$ denotes the generalized $K_{v}^{\rho}$ transform of $f$, then $F(x)$ is infinitely differentiable on $(0, \infty)$ and

$$
\frac{d^{r}}{d x^{r}} F(x)=\left\langle f(t), \frac{\partial^{r}}{\partial x^{r}} Z_{\rho}^{v}(x t)\right\rangle, \quad x>0, r \in \mathbb{N} .
$$

Proof. Consider $h$ an arbitrary increment in $x>0$. Assume, without loss of generality, that $0<|h|<(x / 2)^{\rho /(\rho+1)}$.

It is easy to see that

$$
\frac{F(x+h)-F(h)}{h}=\left\langle f(t), \frac{1}{h}\left(Z_{\rho}^{v}(t(x+h))-Z_{\rho}^{v}(t x)\right)\right\rangle .
$$

We must see that

$$
\varphi_{h}(x, t)=\frac{1}{h}\left(Z_{\rho}^{v}(t(x+h))-Z_{\rho}^{v}(t x)\right)-\frac{\partial}{\partial x} Z_{\rho}^{v}(x t) \longrightarrow 0
$$

as $h \rightarrow 0$, in the sense of the convergence in $\mathbb{E}(I)$, since the result is obtained for $k=1$, by (2.5) and the continuity of $f(t)$.

For every $r \in \mathbb{N}$ we can write

$$
\begin{aligned}
\frac{\partial^{r}}{\partial t^{r}} \varphi_{h}(x, t)= & \frac{1}{h} \int_{x}^{x+h} \int_{x}^{u} \frac{\partial^{2}}{\partial y^{2}}\left(\frac{\partial^{r}}{\partial t^{r}} Z_{\rho}^{v}(t y)\right) d y d u \\
= & \frac{1}{h} \int_{x}^{x+h} \int_{x}^{u} \frac{\partial^{2}}{\partial y^{2}}\left(y^{r} \frac{d^{r}}{d(t y)^{r}} Z_{\rho}^{v}(t y)\right) d y d u \\
= & \frac{1}{h} \int_{x}^{x+h} \int_{x}^{u}\left(r(r-1) y^{r-2} \frac{d^{r}}{d(t y)^{r}} Z_{\rho}^{v}(t y)+2 r y^{r-1} t \frac{d^{r+1}}{d(t y)^{r+1}} Z_{\rho}^{v}(t y)\right. \\
& \left.+y^{r} t^{2} \frac{d^{r+2}}{d(t y)^{r+2}} Z_{\rho}^{v}(t y)\right) d y d u .
\end{aligned}
$$

By virtue of (1.5), given $t \in K, K$ compact, $y>x / 2$, it follows that there exists a positive constant $C$ such that

$$
\left|\frac{d^{r+j}}{d(t y)^{r+j}}\left(Z_{\rho}^{v}(t y)\right)\right| \leq C(y t)^{(2 v-\rho) /(2 \rho+2)} \cdot e^{-(t x / 2)^{\rho /(\rho+1)}}, \quad \text { for } j \in \mathbb{N} .
$$

Therefore

$$
\begin{aligned}
\left|\frac{\partial^{r}}{\partial t^{r}} \varphi_{h}(x, t)\right| \leq & C \cdot t^{(2 v-\rho) /(2 \rho+2)} \cdot e^{-(t x / 2)^{\rho /(\rho+1)}} \\
& \cdot \frac{1}{h} \int_{x}^{x+h} \int_{x}^{u}\left(y^{r-2}+y^{r-1} t+y^{r} t^{2}\right) d y d u \\
\leq & C \cdot e^{-\alpha \cdot(t x)^{\rho /(\rho+1)}}
\end{aligned}
$$

for all $t \in K$ and $0<|h|<(x / 2)^{\rho /(\rho+1)}$, being $\alpha$ a suitable constant. 
Then given $\epsilon>0$, there exists $t_{0} \in K=[a, b]$ such that

$$
\left|\frac{\partial^{r}}{\partial t^{r}} \varphi_{h}(x, t)\right|<\epsilon
$$

for $t>t_{0}$ and $0<|h|<x^{\rho /(\rho+1)} / 2$.

Furthermore, for each $t \in\left[a, t_{0}\right]$ we have

$$
\left|\frac{\partial^{r}}{\partial t^{r}} \varphi_{h}(x, t)\right| \leq C \cdot\left|\frac{1}{h} \int_{x}^{x+h} \int_{x}^{u}\left(y^{r-2}+y^{r-1} t+y^{r} t^{2}\right) d y d u\right| .
$$

Then $\left(\partial^{r} / \partial t^{r}\right) \varphi_{h}(x, t) \rightarrow 0$ as $h \rightarrow 0$ uniformly in $t \in\left[a, t_{0}\right]$. Therefore it is concluded that $\gamma_{r}\left(\varphi_{h}(x, t)\right) \rightarrow 0$ as $h \rightarrow 0$.

By proceeding inductively the result follows.

Proposition 2.3. Let $f \in \mathfrak{E}^{\prime}(I)$ and $F(x)=\left(K_{v}^{\rho} f\right)(x)$ for $x>0$, then

$$
|F(x)| \leq C \cdot x^{r+(2 v-\rho) /(2 \rho+2)} \cdot e^{-\alpha x^{\rho /(\rho+1)}}, \quad x>0,
$$

being $C$ and $\alpha$ suitable constants and $r \in \mathbb{N}$.

Proof. We know by [13, Theorem 1.8-1, pages 18-19] that there exists $r \in \mathbb{N}$ such that

$$
|F(x)| \leq C \max _{0 \leq k \leq r} \gamma_{k}\left(Z_{\rho}^{v}(x t)\right) \quad \forall x>0 .
$$

By the asymptotic behaviour we have for every $K$ compact,

$$
\begin{aligned}
|F(x)| & \leq C \max _{0 \leq k \leq r} \sup _{t \in K}\left|x^{k} \frac{d^{k}}{d(x t)^{k}}\left(Z_{\rho}^{v}(x t)\right)\right| \\
& \leq C \max _{0 \leq k \leq r} \sup _{t \in K}\left|x^{k}(x t)^{(2 v-\rho) /(2 \rho+2)} e^{-(x t)^{\rho /(\rho+1)}}\right| \\
& \leq C \cdot x^{r+(2 v-\rho) /(2 \rho+2)} \cdot \max _{0 \leq k \leq r} \sup _{t \in K}\left|t^{(2 v-\rho) /(2 \rho+2)} e^{-(x t)^{\rho /(\rho+1)}}\right| \\
& \leq C \cdot x^{r+(2 v-\rho) /(2 \rho+2)} e^{-\alpha x^{\rho /(\rho+1)}} .
\end{aligned}
$$

With the following proposition we obtain an operational formula for the generalized $K_{v}^{\rho}$ transform, that includes the operator $A_{\rho, v}^{*}, \rho \in \mathbb{N}$.

Proposition 2.4. Let $P$ be a polynomial, if $f \in \mathfrak{E}^{\prime}(I)$, then

$$
\left(K_{v}^{\rho} P\left(A_{\rho, v}^{*}\right) f\right)(x)=P\left((-x)^{\rho}\right)\left(K_{v}^{\rho} f\right)(x)
$$

for $x>0$ and $\rho \in \mathbb{N}$.

Proof. By (1.6) and according to Proposition 2.1 it follows that

$$
\begin{aligned}
\left(K_{v}^{\rho} P\left(A_{\rho, v}^{*}\right) f\right)(x) & =\left\langle P\left(A_{\rho, v}^{*}\right) f(t), Z_{\rho}^{v}(x t)\right\rangle \\
& =\left\langle f(t), P\left(A_{\rho, v}\right) Z_{\rho}^{v}(x t)\right\rangle \\
& =\left\langle f(t), P\left((-x)^{\rho}\right) Z_{\rho}^{v}(x t)\right\rangle \\
& =P\left((-x)^{\rho}\right)\left(K_{v}^{\rho} f\right)(x) .
\end{aligned}
$$


Now we establish an inversion theorem for the $K_{v}^{\rho}$ - transform using a similar procedure to employ by Malgonde and Saxena [7].

LEMMA 2.5. Let $f \in \mathfrak{E}^{\prime}(I)$. Then

$$
\int_{0}^{\infty} x^{-s}\left\langle f(t), Z_{\rho}^{v}(x t)\right\rangle d x=\left\langle f(t), \int_{0}^{\infty} x^{-s} Z_{\rho}^{v}(x t) d x\right\rangle .
$$

Proof. Let $N \in \mathbb{N}$. First, we prove that

$$
\int_{0}^{N} x^{-s}\left\langle f(t), Z_{\rho}^{v}(x t)\right\rangle d x=\left\langle f(t), \int_{0}^{N} x^{-s} Z_{\rho}^{v}(x t) d x\right\rangle .
$$

We take $\left\{x_{r, l}\right\}_{r=0}^{l}$ a partition of the interval $[0, N]$ such that $d_{l}=x_{r, l}-x_{r-1, l}$ for each $r=1,2, \ldots, l$. Then we can write

$$
\begin{aligned}
\int_{0}^{N} x^{-s}\left\langle f(t), Z_{\rho}^{v}(x t)\right\rangle d x & =\lim _{l \rightarrow \infty} d_{l} \cdot \sum_{r=0}^{l} x_{r, l}^{-s}\left\langle f(t), Z_{\rho}^{v}\left(x_{r, l} t\right)\right\rangle, \\
d_{l} \cdot \sum_{r=0}^{l} x_{r, l}^{-s}\left\langle f(t), Z_{\rho}^{v}\left(x_{r, l} t\right)\right\rangle & =\left\langle f(t), d_{l} \sum_{r=0}^{l} x_{r, l}^{-s} Z_{\rho}^{v}\left(x_{r, l} t\right)\right\rangle .
\end{aligned}
$$

Then (2.18) is established if we demonstrated

$$
\lim _{l \rightarrow \infty} d_{l} \sum_{r=0}^{l} x_{r, l}^{-s} \cdot Z_{\rho}^{v}\left(x_{r, l} t\right)=\int_{0}^{N} x^{-s} Z_{\rho}^{v}(x t) d x
$$

in the sense of the convergence in $\mathbb{E}(I)$.

Then, since the function

$$
g(t, x)= \begin{cases}x^{-s+k} \frac{d^{k}}{d(x t)^{k}}\left(Z_{\rho}^{v}(x t)\right) & \text { if } x \in[0, N] \\ 0 & \text { if } x=0\end{cases}
$$

with $t \in[a, b], 0<a<b<\infty$, is uniformly continuous on $(t, x) \in[a, b] \times[0, N]$, we have that if $\epsilon>0$ and $m \in \mathbb{N}$, there exists $l_{0} \in \mathbb{N}$ such that

$$
\sup _{t \in K}\left|\frac{d^{k}}{d t^{k}}\left[d_{l} \sum_{r=0}^{l} x_{r, l}^{-s} Z_{\rho}^{v}\left(x_{r, l} t\right)-\int_{0}^{N} x^{-s} Z_{\rho}^{v}(x t) d x\right]\right|<\epsilon
$$

for $l>l_{0}$. Therefore we obtain (2.20).

Using (1.5), for $m, k \in \mathbb{N}$, we have that

$$
\left|\frac{d^{k}}{d t^{k}} \int_{N}^{\infty} x^{-s} Z_{\rho}^{v}(x t) d x\right| \leq C \cdot t^{(2 v-\rho) /(2 \rho+2)} \int_{N}^{\infty} x^{-\operatorname{Re} s+(2 v-\rho) /(2 \rho+2)} e^{-(x t)^{\rho /(\rho+1)}} d x \rightarrow 0
$$

uniformly in $t \in K$, as $N \rightarrow \infty$.

Moreover, by Proposition 2.3 we have

$$
\left|\int_{N}^{\infty} x^{-s}\left\langle f(t), Z_{\rho}^{v}(x t)\right\rangle d x\right| \leq C \int_{N}^{\infty} x^{-s+r+(2 v-\rho) /(2 \rho+2)} e^{-\alpha x^{\rho /(\rho+1)}} d x,
$$

being $\alpha$ a suitable constant. 
Then

$$
\lim _{N \rightarrow \infty} \int_{N}^{\infty} x^{-s}\left\langle f(t), Z_{\rho}^{v}(x t)\right\rangle d x=0 .
$$

Hence by (2.18), (2.23), and (2.25) we obtain (2.17).

The following lemma is obtained from [7, Lemmas 2 and 3] with a slight modification.

LEMMA 2.6. Let $\phi \in \mathbb{D}(I)$, we denote

$$
\psi(s)=\int_{0}^{\infty} y^{-s} \phi(y) d y .
$$

Then

(i) $\int_{-R}^{R}\left\langle f(u), u^{\sigma+i w-1}\right\rangle \psi(\sigma+i w) d w=\left\langle f(u), \int_{-R}^{R} u^{\sigma+i w-1} \psi(\sigma+i w) d w\right\rangle$ with $\sigma>0$ and $R>0$,

(ii) $(1 / \pi) \int_{0}^{\infty} \phi(y)(u / y)^{\sigma}(\sin (R \log (u / y)) / u \log (u / y)) d y \rightarrow \phi(u)$ as $R \rightarrow \infty$, in the sense of the convergence in $\mathbb{E}(I)$, with $\sigma>0$.

Now we demonstrate the first inversion theorem.

THEOREM 2.7. Let $f \in \mathbb{E}^{\prime}(I), \phi \in \mathbb{D}(I), v \in \mathbb{C}$ and $\sigma<1+\min (0, \operatorname{Re} v)$. Then

$$
\lim _{R \rightarrow \infty}\left\langle\frac{1}{2 \pi i} \int_{\sigma-i R}^{\sigma+i R} \frac{1}{K(s)} y^{-s} \int_{0}^{\infty} x^{-s} F(x) d x d s, \phi(y)\right\rangle=\langle f(t), \phi(t)\rangle,
$$

where $F(x)=\left(K_{v}^{\rho} f\right)(x)$, for $x>0$, and

$$
K(s)=\frac{1}{\rho} \Gamma(1-s) \Gamma\left(\frac{1-s+v}{\rho}\right) .
$$

Proof. Given $f \in \mathfrak{E}^{\prime}(I)$, we denote $F(x)=\left\langle f(t), Z_{\rho}^{v}(x t)\right\rangle$, for $x>0$. It is easy to see that the function

$$
\varphi_{R}(y)=\frac{1}{2 \pi i} \int_{\sigma-i R}^{\sigma+i R} \frac{1}{K(s)} y^{-s} \int_{0}^{\infty} x^{-s} F(x) d x d s
$$

is continuous for $y>0, R>0$. Therefore, $\varphi_{R}(y)$ defines a regular distribution in ¿') $^{\prime}(I)$ being

$$
\left\langle\varphi_{R}(y), \phi(y)\right\rangle=\frac{1}{2 \pi i} \int_{0}^{\infty} \phi(y) \int_{\sigma-i R}^{\sigma+i R} \frac{1}{K(s)} y^{-s} \int_{0}^{\infty} x^{-s} F(x) d x d s d y
$$

for all $\phi \in \mathfrak{D}(I)$.

By the Fubini's theorem we can interchange the order of integration and we can write

$$
\left\langle\varphi_{R}(y), \phi(y)\right\rangle=\frac{1}{2 \pi i} \int_{\sigma-i R}^{\sigma+i R} \frac{1}{K(s)}\left[\int_{0}^{\infty} x^{-s}\left\langle f(t), Z_{\rho}^{v}(x t)\right\rangle d x\right] \int_{0}^{\infty} y^{-s} \phi(y) d y d s
$$


By Lemma 2.5, we get

$$
\begin{aligned}
\left\langle\varphi_{R}(y), \phi(y)\right\rangle & =\frac{1}{2 \pi i} \int_{\sigma-i R}^{\sigma+i R} \frac{1}{K(s)}\left\langle f(t), t^{s-1} \int_{0}^{\infty} u^{-s} Z_{\rho}^{v}(u) d u\right\rangle \int_{0}^{\infty} y^{-s} \phi(y) d y d s \\
& =\frac{1}{2 \pi i} \int_{\sigma-i R}^{\sigma+i R}\left\langle f(t), t^{s-1}\right\rangle \int_{0}^{\infty} y^{-s} \phi(y) d y d s .
\end{aligned}
$$

Then Lemma 2.6(i) permits us to obtain

$$
\left\langle\varphi_{R}(y), \phi(y)\right\rangle=\left\langle f(t), \frac{1}{2 \pi i} \int_{\sigma-i R}^{\sigma+i R} t^{s-1} \int_{0}^{\infty} y^{-s} \phi(y) d y d s\right\rangle .
$$

Finally, interchanging the order of integration and using Lemma 2.6(ii) we achieve

$$
\left.\left\langle\varphi_{R}(y), \phi(y)\right\rangle=\left\langle f(t), \frac{1}{\pi} \int_{0}^{\infty}\left(\frac{u}{y}\right)^{\sigma} \phi(y) \frac{\sin (R \log (u / y))}{u \log (u / y)} d y\right\rangle \rightarrow\langle f(t), \phi(t)\rangle\right\rangle
$$

as $R \rightarrow \infty$. With this, Theorem 2.7 is demonstrated.

By Theorem 2.7 the following uniqueness theorem is deduced.

THEOREM 2.8. Let $f, g \in \mathfrak{E}^{\prime}(I)$ and $v \in \mathbb{C}$. If $\left(K_{v}^{\rho} f\right)(x)=\left(K_{v}^{\rho} g\right)(x)$, for $x>0$, then $f=g$ in the sense of an equality in $\mathbb{B}^{\prime}(I)$.

Proof. It is sufficient to see that for each $\phi \in \mathbb{D}(I),\langle f(t)-g(t), \phi(t)\rangle=0$.

This fact is established immediately since,

$$
\begin{aligned}
\langle f(t) & -g(t), \phi(t)\rangle \\
= & \left\langle\lim _{R \rightarrow \infty} \frac{1}{2 \pi i} \int_{\sigma-i R}^{\sigma+i R} \frac{1}{K(s)} y^{-s} \int_{0}^{\infty} x^{-s}\left(\left(K_{v}^{\rho} f\right)(x)-\left(K_{v}^{\rho} g\right)(x)\right) d x d s, \phi(y)\right\rangle=0,
\end{aligned}
$$

where $\sigma$ and $K(s)$ are as in Theorem 2.7.

In the following theorem we obtain another inversion formula, in which appears a generalization of an operator of Post-Widder type, being obtained as a particular case an inversion formula for the Laplace and Meijer transformation.

THEOREM 2.9. Let $f \in \mathfrak{E}^{\prime}(I)$ and $\operatorname{Re} v \geq \rho / 2-1$. If $F(x)$ denotes the $K_{v}^{\rho}$ generalized transform of $f$, then

$$
\lim _{k \rightarrow \infty}\left\langle B_{\rho, v, k}^{*} F\left(\frac{\rho k}{y}\right), \phi(y)\right\rangle=\langle f(t), \phi(t)\rangle
$$

for each $\phi \in \mathbb{D}(I)$, where $B_{\rho, v, k}^{*}$ is the adjoint operator of $B_{\rho, v, k}$.

Proof. Let $f \in \mathfrak{E}^{\prime}(I)$ and $F(x)=\left\langle f(t), Z_{\rho}^{v}(x t)\right\rangle$, for $x>0$.

By Proposition 2.1 and (1.6) we have

$$
\begin{aligned}
B_{\rho, v, k}^{*} F\left(\frac{\rho k}{y}\right) & =C_{1} y^{-\rho k-1}\left\langle f(t), B_{v, \rho}^{k} Z_{\rho}^{v}\left(\frac{\rho k t}{y}\right)\right\rangle \\
& =C_{1} y^{-\rho k-1}\left\langle f(t), \rho^{k}(\rho k t)^{\rho k} Z_{\rho}^{v}\left(\frac{\rho k t}{y}\right)\right\rangle,
\end{aligned}
$$

$C_{1}$ being introduced in Section 1. 
Moreover, $B_{\rho, v, k}^{*} F(\rho k / y)$ defines a regular distribution in $\mathbb{D}^{\prime}(I)$ by

$$
\left\langle B_{\rho, v, k}^{*} F\left(\frac{\rho k}{y}\right), \phi(y)\right\rangle=\int_{0}^{\infty} B_{\rho, v, k}^{*} F\left(\frac{\rho k}{y}\right) \phi(y) d y, \quad \forall \phi \in \mathbb{D}(I) .
$$

We must see that

$$
\left\langle B_{\rho, v, k}^{*} F\left(\frac{\rho k}{y}\right), \phi(y)\right\rangle=\left\langle f(t), C_{1} t^{\rho k} \int_{0}^{\infty} y^{-\rho k-1} Z_{\rho}^{v}\left(\frac{\rho k t}{y}\right) \phi(y) d y\right\rangle
$$

for every $\phi \in \mathbb{D}(I)$.

Consider $0<a<b<\infty$ such that $\phi(x)=0, x \notin[a, b], \phi \in \mathbb{D}(I)$.

We denote by $\left\{y_{m, l}\right\}_{m=0}^{l}$ a partition of $[a, b]$ with $d_{l}=y_{m, l}-y_{m, l-1}, m=1,2, \ldots, l$, then we achieve

$$
\begin{aligned}
\int_{0}^{\infty} B_{\rho, v, k}^{*} F & \left(\frac{\rho k}{y}\right) \phi(y) d y \\
& =\lim _{l \rightarrow \infty} d_{l} \sum_{m=0}^{l} B_{\rho, v, k}^{*} F\left(\frac{\rho k}{y_{m, l}}\right) \phi\left(x_{m, l}\right) \\
& =\lim _{l \rightarrow \infty}\left\langle f(t), t^{\rho k} C_{1} \rho^{k}(\rho k)^{\rho k} d_{l} \sum_{m=0}^{l} y_{m, l}^{-\rho k-1} Z_{\rho}^{v}\left(\frac{\rho k t}{y_{m, l}}\right) \phi\left(x_{m, l}\right)\right\rangle .
\end{aligned}
$$

Therefore we must see that

$$
\lim _{l \rightarrow \infty} d_{l} \sum_{m=0}^{l} y_{m, l}^{-\rho k-1} Z_{\rho}^{v}\left(\frac{\rho k t}{y_{m, l}}\right) \phi\left(y_{m, l}\right)=\int_{a}^{b} y^{-\rho k-1} Z_{\rho}^{v}\left(\frac{\rho k t}{y}\right) \phi(y) d y
$$

in the sense of the convergence in $\mathbb{E}(I)$.

Consider $K \subset(0, \infty)$ compact and $r \in \mathbb{N}$.

Then we have

$$
\begin{aligned}
& \frac{d^{r}}{d t^{r}}\left(d_{l} \sum_{m=0}^{l} y_{m, l}^{-\rho k-1} Z_{\rho}^{v}\left(\frac{\rho k t}{y_{m, l}}\right) \phi\left(y_{m, l}\right)-\int_{a}^{b} y^{-\rho k-1} Z_{\rho}^{v}\left(\frac{\rho k t}{y}\right) \phi(y) d y\right) \\
& \quad=d_{l} \sum_{m=0}^{l} y_{m, l}^{-\rho k-1} \frac{d^{r}}{d t^{r}}\left(Z_{\rho}^{v}\left(\frac{\rho k t}{y_{m, l}}\right)\right) \phi\left(y_{m, l}\right)-\int_{a}^{b} y^{-\rho k-1} \frac{d^{r}}{d t^{r}}\left(Z_{\rho}^{v}\left(\frac{\rho k t}{y}\right)\right) \phi(y) d y .
\end{aligned}
$$

Hence, since the function $y^{-\rho k-1}\left(d^{r} / d t^{r}\right)\left(Z_{\rho}^{v}(\rho k t / y)\right) \phi(y)$ is uniformly continuous for $(x, y) \in[a, b] \times K$, then

$$
\lim _{l \rightarrow \infty} d_{l} \sum_{m=0}^{l} y_{m, l}^{-\rho k-1} Z_{\rho}^{v}\left(\frac{\rho k t}{y_{m, l}}\right) \phi\left(x_{m, l}\right)=\int_{a}^{b} y^{-\rho k-1} Z_{\rho}^{v}\left(\frac{\rho k t}{y}\right) \phi(y) d y
$$

uniformly in $y \in K$. Thus (2.39) is demonstrated. 
On the other hand, making a change of variables we obtain

$$
\int_{0}^{\infty} B_{\rho, v, k}^{*} F\left(\frac{\rho k}{y}\right) \phi(y) d y=\left\langle g(t), C_{1} t^{-\rho k-1} \int_{0}^{\infty} u^{\rho k} Z_{\rho}^{v}\left(\frac{\rho k u}{t}\right) \psi(u) d u\right\rangle
$$

with $g(t)=(1 / t) f(1 / t)$ and $\psi(u)=(1 / u) \phi(1 / u)$.

To complete the demonstration we see that

$$
\lim _{k \rightarrow \infty} C_{1} t^{-\rho k-1} \int_{0}^{\infty} u^{\rho k} Z_{\rho}^{v}\left(\frac{\rho k u}{t}\right) \psi(u) d u=\psi(t)
$$

in the sense of the convergence in $\mathbb{E}(I)$.

Now, according to (1.9) we have

$$
\begin{aligned}
\int_{0}^{\infty} u^{\rho k-((2 v-\rho) /(2 \rho+2))-(1 /(\rho+1))} Z_{\rho}^{v}\left(\frac{\rho k u}{t}\right) d u \\
=\left(\frac{t}{\rho k}\right)^{(\rho+1) k-((2 v-\rho) /(2 \rho+2))-(1 /(\rho+1))+1} \rho^{-1} \Gamma\left(\rho k-\frac{2 v-\rho}{2 \rho+2}-\frac{1}{\rho+1}+1\right) \\
\quad \cdot \Gamma\left(\frac{\rho k-((2 v-\rho) /(2 \rho+2))-(1 /(\rho+1))+1+v}{\rho}\right) .
\end{aligned}
$$

Then, we can deduce that

$$
\begin{aligned}
\frac{d^{r}}{d t^{r}} & \left(C_{1} t^{-\rho k-1} \int_{0}^{\infty} u^{\rho k} Z_{\rho}^{v}\left(\frac{\rho k u}{t}\right) \psi(u) d u-\psi(t)\right) \\
= & C_{1} t^{-\rho k-1} \int_{0}^{\infty} u^{\rho k-((2 v-\rho) /(2 \rho+2))-(1 /(\rho+1))} Z_{\rho}^{v}\left(\frac{\rho k u}{t}\right) \\
& \cdot\left(u^{((2 v-\rho) /(2 \rho+2))+(1 /(\rho+1))}\left(\frac{u}{t} \frac{d}{d u}\right)^{r} \psi(u)-t^{((2 v-\rho) /(2 \rho+2))+(1 /(\rho+1))} \frac{d^{r}}{d t^{r}} \psi(t)\right) d u
\end{aligned}
$$

for every $r \in \mathbb{N}$.

Therefore by (1.5) we have that for all $t \in K, K \subset(0, \infty), K$ compact, there exists a constant $C>0$ such that

$$
\begin{aligned}
\mid \frac{d^{r}}{d t^{r}} & \left(C \cdot t^{-(\rho+1) k-1} \int_{0}^{\infty} Z_{\rho}^{v}\left(\frac{\rho k t}{y}\right) z^{(\rho+1) k} \psi(z) d z-\psi(t)\right) \mid \\
\leq & C \frac{(\rho k)^{\rho k-k+1-1 /(\rho+1)}}{((\rho+1) k) !} t^{-\rho k-\rho /(\rho+1)} \frac{\rho}{\rho+1} \int_{0}^{\infty} z^{(\rho+1) k} e^{-\alpha_{2}(\rho k z / t)} \\
& \cdot\left|z^{(2 v-\rho) /(2 \rho+2)}\left(\frac{z}{t} \frac{d}{d z}\right)^{r} \psi(z)-t^{(2 v-\rho) /(2 \rho+2)} \frac{d^{r}}{d t^{r}} \psi(t)\right| d z .
\end{aligned}
$$

We divided the last integral in this way

$$
\begin{array}{r}
C_{1} \int_{0}^{\infty}=C_{1}\left(\int_{0}^{((\rho+1) k)^{1 / \rho}(1-\eta)^{(\rho+1) / \rho} t(\rho k)^{-1}}+\int_{((\rho+1) k)^{1 / \rho}(1-\eta)^{(\rho+1) / \rho} t(\rho k)^{-1}}^{((\rho+1) k)^{1 / \rho}(1+\eta)^{(\rho+1) / \rho} t(\rho k)^{-1}}\right. \\
\left.+\int_{((\rho+1) k)^{1 / \rho}(1+\eta)^{(\rho+1) / \rho} t(\rho k)^{-1}}^{\infty}\right)=I_{1}(t, k)+I_{2}(t, k)+I_{3}(t, k)
\end{array}
$$

with $\eta>0$ and $C_{1}=C\left((\rho k)^{\rho k-k+1-1 /(\rho+1)} /((\rho+1) k) !\right) t^{-\rho k-\rho /(\rho+1)} \rho /(\rho+1)$. 
For each $t \in K$, we obtain

$$
\begin{aligned}
& \left|I_{1}(t, k)\right| \\
& \leq C \frac{(\rho k)^{\rho k-k+1-1 /(\rho+1)}}{((\rho+1) k) !} t^{-\rho k-\rho /(\rho+1)} \frac{\rho}{\rho+1} \\
& \quad \cdot\left(\int_{0}^{((\rho+1) k)^{1 / \rho}(1-\eta)^{(\rho+1) / \rho} t(\rho k)^{-1}} u^{\rho k+(2 v-\rho) /(2 \rho+2)} e^{-(\rho k u / t)^{\rho /(\rho+1)}}\left|\left(\frac{u}{t} \frac{d}{d u}\right)^{r} \psi(u)\right| d u\right. \\
& \quad+\int_{0}^{((\rho+1) k)^{1 / \rho}(1-\eta)^{(\rho+1) / \rho} t(\rho k)^{-1}} u^{\rho k-1 /(\rho+1)} e^{-(\rho k u / t)^{\rho /(\rho+1)}} \\
& \left.\quad \cdot t^{(2 v-\rho) /(2 \rho+2)+1 /(\rho+1)}\left|\frac{d^{r}}{d t^{r}} \psi(t)\right| d u\right)
\end{aligned}
$$

and making an appropriate change of variables, we obtain

$$
\left|I_{1}(t, k)\right| \leq C \frac{((\rho+1) k)^{(\rho+1) k+1}}{((\rho+1) k) !} \int_{0}^{1-\eta} x^{(\rho+1) k} e^{-(\rho+1) k x} d x
$$

$C$ being a suitable constant and $\operatorname{Re} v \geq \rho / 2-1$.

Now, using [10, Theorem (5b), page 288] we achieve

$$
I_{1}(t, k) \longrightarrow 0
$$

as $k \rightarrow \infty$ uniformly in $t \in K$.

Proceeding in a similar way we obtain that

$$
I_{3}(t, k) \longrightarrow 0
$$

as $k \rightarrow \infty$ uniformly in $t \in K$.

Then, it remains $I_{2}(t, k)$, for this, using the mean value theorem we can write

$$
\left|u^{(2 v-\rho) /(2 \rho+2)+1 /(\rho+1)}\left(\frac{u}{t} \frac{d}{d u}\right)^{r} \psi(u)-t^{(2 v-\rho) /(2 \rho+2)+1 /(\rho+1)} \frac{d^{r}}{d t^{r}} \psi(t)\right| \leq C_{2}|t-u|
$$

for $u, t \in(0, \infty)$ and $C_{2}$ a suitable constant.

Then if $u \in\left(((\rho+1) k)^{1 / \rho}(1-\eta)^{(\rho+1) / \rho} t(\rho k)^{-1},((\rho+1) k)^{1 / \rho}(1+\eta)^{(\rho+1) / \rho} t(\rho k)^{-1}\right)$ we have

$$
\left|I_{2}(t, k)\right| \leq C_{3} \frac{((\rho+1) k)^{(\rho+1) k+1}}{((\rho+1) k) !} \int_{1-\eta}^{1+\eta} x^{(\rho+1) k} e^{-(\rho+1) k x} d x,
$$

applying [10, Theorem (5b), page 287] we obtain the desired result.

Therefore we can deduce that

$$
\lim _{k \rightarrow \infty}\left\langle C_{\rho, v, k}^{*} F\left(\frac{\rho k}{y}\right), \phi(y)\right\rangle=\langle g(t), \psi(t)\rangle=\langle f(t), \phi(t)\rangle \quad \forall \phi \in \mathbb{D}(I) .
$$




\section{REFERENCES}

[1] B. Bonilla, M. Rivero, J. Rodríguez, J. Trujillo, and A. A. Kilbas, Bessel-type functions and Bessel-type integral transforms on spaces $\mathfrak{f}_{p, \mu}$ and $\mathfrak{f}_{p, \mu}^{\prime}$, Integral Transform. Spec. Funct. 8 (1999), no. 1-2, 13-30. CMP 1730 610. Zbl 937.33004.

[2] D. I. Cruz and J. Rodríguez, A weighted norm inequality for a generalization of the $K$ transform, J. Inst. Math. Comput. Sci. Comput. Sci. Ser. 9 (1998), no. 1, 25-29. CMP 1702625.

[3] _ Abelian and Tauberian theorems for the Krätzel transform, Pure Appl. Math. Sci. 49 (1999), no. 1-2, 45-60. MR 2000d:44003.

[4] S. A. Emara and H. P. Heinig, Weighted norm inequalities for the Hankel- and $K$ transformations, Proc. Roy. Soc. Edinburgh Sect. A 103 (1986), no. 3-4, 325-333. MR 88f:44002. Zbl 622.44004.

[5] E. Krätzel, Integral transformations of Bessel-type, Generalized Functions and Operational Calculus (Proc. Conf., Varna, 1975), Bulgar. Acad. Sci., Sofia, 1979, pp. 148-155. MR 80m:44015. Zbl 403.44003.

[6] E. Krätzel and H. Menzer, Verallgemeinerte Hankel-funktionen, Publ. Math. Debrecen 18 (1971), 139-147 (1972) (German). MR 46\#9410. Zbl 247.33014.

[7] S. P. Malgonde and R. K. Saxena, An inversion formula for the distributional $\mathrm{H}$ transformation, Math. Ann. 258 (1981/82), no. 4, 409-417. MR 83e:44002. Zbl 486.46032.

[8] G. L. N. Rao and L. Debnath, A generalized Meijer transformation, Int. J. Math. Math. Sci. 8 (1985), no. 2, 359-365. MR 87k:46091. Zbl 597.46036.

[9] L. Schwartz, Théorie des Distributions, Publications de l'Institut de Mathematique de l'Universite de Strasbourg, no. 9-10. Nouvelle edition, entierement corrigee, refondue et augmentee, Hermann, Paris, 1966 (French). MR 35\#730. Zbl 149.09501.

[10] D. V. Widder, The Laplace Transform, Princeton Mathematical Series, vol. 6, Princeton University Press, Princeton, NJ, 1941. MR 3,232d. Zbl 063.08245.

[11] A. H. Zemanian, Distribution Theory and Transform Analysis. An Introduction to Generalized Functions, with Applications, McGraw-Hill Book Co., New York, Toronto, London, Sydney, 1965. MR 31\#1556. Zbl 127.07201.

[12] _ A distributional K transformation, SIAM J. Appl. Math. 14 (1966), 1350-1365; errata, ibid. 15 (1966), 765. MR 35\#2094. Zbl 154.13902.

[13] _ Generalized Integral Transformations, Pure and Applied Mathematics, vol. 18, Interscience Publishers [John Wiley \& Sons, Inc.], New York, London, Sydney, 1968. MR 54\#10991. Zbl 181.12701.

JosÉ RodRÍGUEz EXPÓSITO: DEPARTAMENTO DE ANÁLISIS MATEMÁTICO, UNIVERSIDAD DE LA LAGUNA, 38271 LA LAGUNA TENERIFE, SPAIN

E-mail address: jorodriguez@u11.es

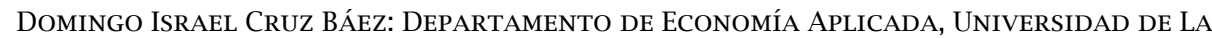
LAGUNA, 38071 LA LAGUNA TENERIFE, SPAIN

E-mail address: dicruz@u11.es 


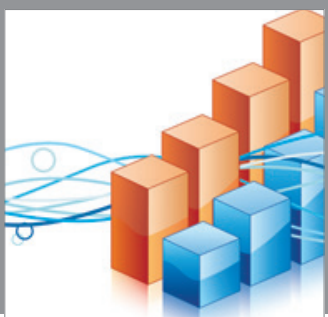

Advances in

Operations Research

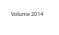

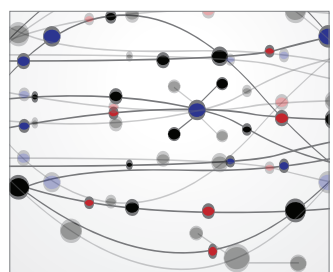

\section{The Scientific} World Journal
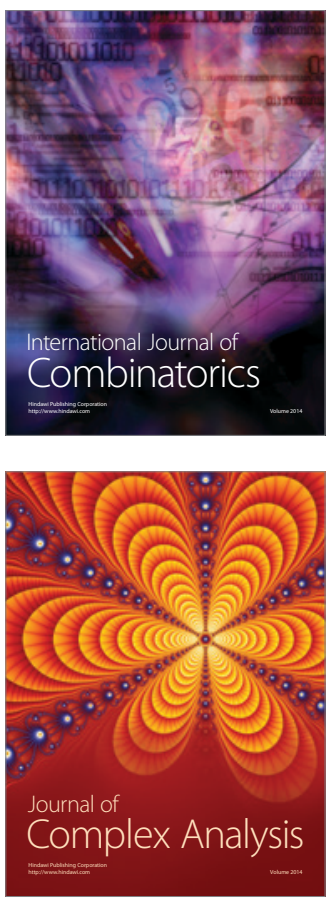

International Journal of

Mathematics and

Mathematical

Sciences
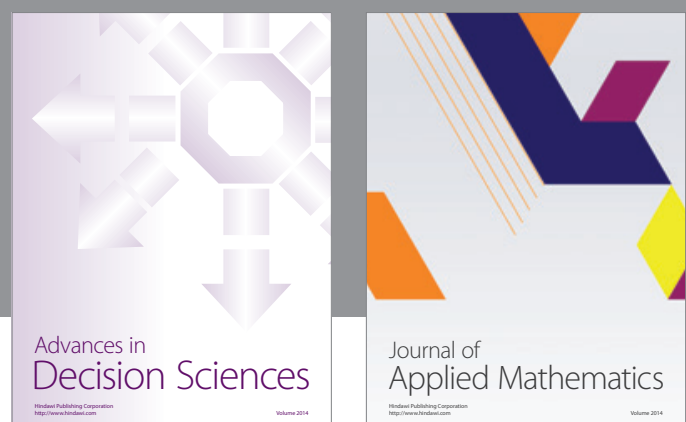

Journal of

Applied Mathematics
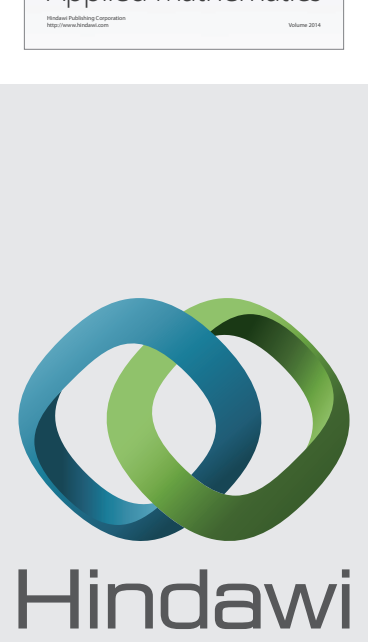

Submit your manuscripts at http://www.hindawi.com
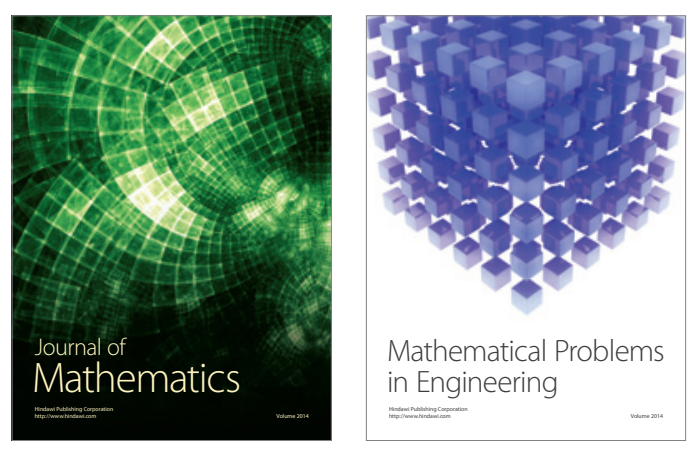

Mathematical Problems in Engineering
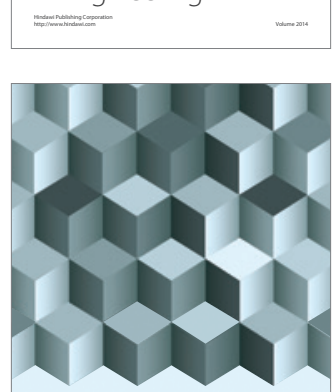

Journal of

Function Spaces
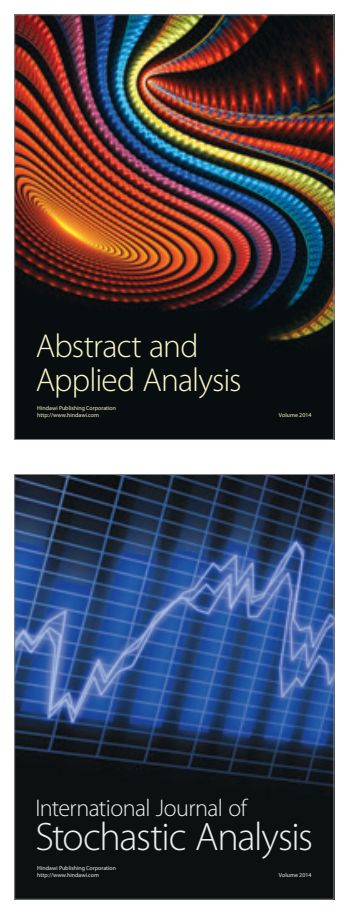

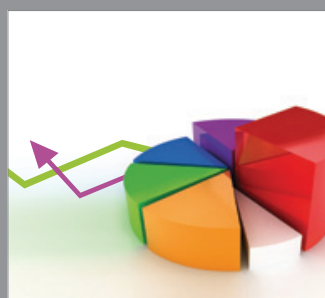

ournal of

Probability and Statistics

Promensencen
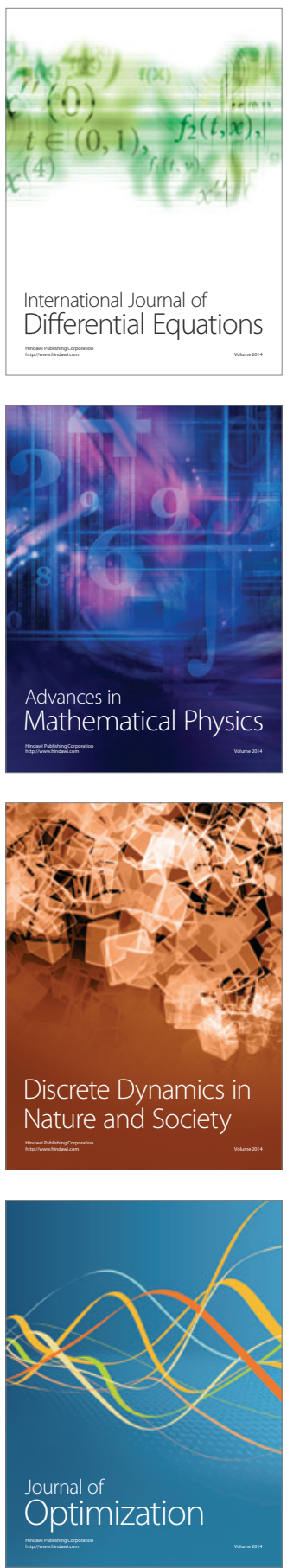